\title{
Individual tooth region segmentation using modified watershed algorithm with morphological characteristic
}

\author{
Sung Dae $\mathrm{Na}^{\mathrm{a}}$, GihyounLee ${ }^{\mathrm{a}}$, Jyung Hyun Lee ${ }^{\mathrm{b}}$ and Myoung Nam Kim ${ }^{\mathrm{c}, *}$ \\ ${ }^{a}$ Department of Medical \& Biological Engineering, Graduate School, Kyungpook National University, \\ Daegu, Republic of Korea \\ ${ }^{b}$ Department of Biomedical Engineering, Kyungpook National University Hospital, Daegu, Republic \\ of Korea \\ ${ }^{c}$ Department of Biomedical Engineering, School of Medicine, Kyungpook National University, Daegu, \\ Republic of Korea
}

\begin{abstract}
In this paper, a new method for individual tooth segmentation was proposed. The proposed method is composed of enhancement and extraction of boundary and seed of watershed algorithm using trisection areas by morphological characteristic of teeth. The watershed algorithm is one of the conventional methods for tooth segmentation; however, the method has some problems. First, molar region detection ratio is reduced because of oral structure features that is low intensities in molar region. Second, inaccurate segmentation occurs in incisor region owing to specular reflection. To solve the problems, the trisection method using morphological characteristic was proposed, where three tooth areas are made using ratio of entire tooth to each tooth. Moreover, the enhancement is to improve the intensity of molar using the proposed method. In addition, boundary and seed of watershed are extracted using trisection areas applied other parameters each area. Finally, individual tooth segmentation was performed using extracted boundary and seed. Furthermore, the proposed method was compared with conventional methods to confirm its efficiency. As a result, the proposed method was demonstrated to have higher detection ratio, better over segmentation, and overlap segmentation than conventional methods.
\end{abstract}

Keywords: Segmentation, tooth, watershed, oral scanner

\section{Introduction}

Tooth healthcare quality has increased recently because of graying improvements and a higher standard of living. Existing problems in tooth healthcare are related to the healing site for oral disease, obstacles, and aesthetics. Prosthetics is an aspect of tooth healthcare that requires considerable time and high cost throughout the process, from impression acquisition to final manufacture [1,2]. Therefore, researchers attempt to address these problems with an image-based intra-oral-scanner [3-5]. In addition, tooth region detection requires research into dental optics-image devices, and accurate detection

\footnotetext{
${ }^{*}$ Corresponding author: Myoung Nam Kim, Department of Biomedical Engineering, School of Medicine, Kyungpook National University, Daegu, Republic of Korea. Tel.: +82-53-200-5266; Fax:+82-53-200-5364; E-mail: kimmn@knu.ac.kr.
}

0959-2989/14/\$27.50 @ 2014 - IOS Press and the authors. 


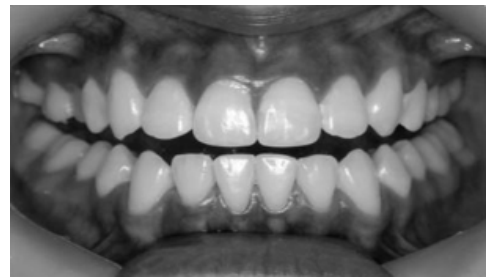

(a)

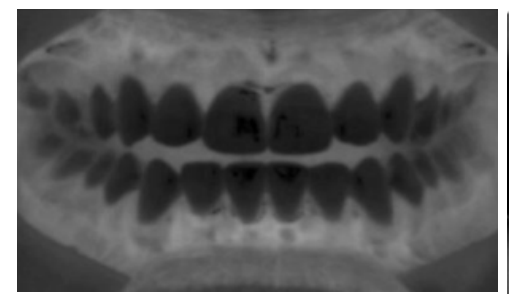

(c)

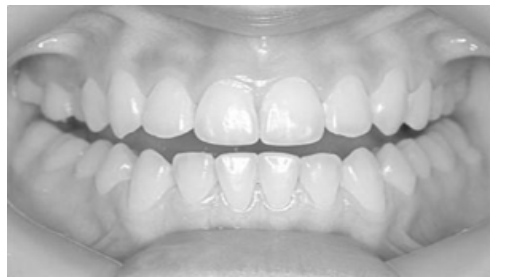

(b)

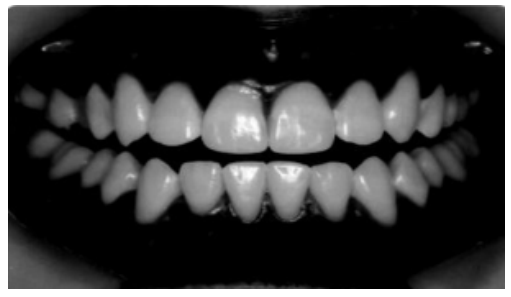

(d)

Fig. 1. Preprocessing result, (a) G of RGB space image, (b) R of RGB space image, (c) tooth complement image and (d) tooth emphatic image.

of the tooth image requires that there should be no over-or overlap-segmentation from the incisor to the molar. Watershed algorithm is a suitable conventional method to segment the individual tooth regions. However, a detection ratio of watershed algorithm was reduced because of the image intensity in the molar region, both the tooth detection ratio and the accuracy decrease owing to the use of Sobel's edge and the regional maximum method [6]. In order to solve the problems, the trisection method of entire tooth area using the morphological characteristic of teeth was proposed. Moreover, the boundary and seed of watershed are made using trisection areas applied each parameters to overcome the reduced detection ratio and inaccurate rate.

The proposed methods have the following steps. First, preprocessing was performed to remove unnecessary parts of the gingival and muscles around the tooth, except for the tooth on the color tooth image. Second, three tooth areas are made using morphological characteristic of teeth to extract the regions of interest. Third, image enhancement was performed to improve the intensity in the molar region using the proposed method. Next, watershed seed and boundary are extracted using trisection areas applied other parameters on each areas. Finally, the watershed algorithm using the acquired seed and boundary perform the individual tooth segmentation.

\section{Method}

\subsection{Preprocessing}

Emphatic tooth image was obtained in preprocessing using the RGB (red, green, blue) space from the difference on the gingival and oral tooth muscle. The tooth regions similarly appear on a histogram of the R, G, and B on the RGB space; however, a histogram of the R intensity appears, except for tooth regions. In summary, the complement and emphatic images of the tooth were acquired using the RGB space difference. Figure 1 shows the results of the experiment using the difference in the RGB space [6]. 


\subsection{Proposed trisection method using morphological characteristic and enhancement}

The intensities of molar regions are low on the tooth image because of the tooth structure. Different intensities cause reductions in tooth detection. In order to improve the intensities, the trisection method was utilized to divide tooth area according to teeth width.

The proposed trisection method has the following steps. First, binary image from the acquired emphatic tooth image in the preprocessing was made with the applied threshold of 25 . Then, unnecessary information was removed from the rest of the image using component size. Second, four pixels are extracted using the acquired binary image, and entire tooth region is made to connect the pixels. Eq. (1) presents the relation of the entire tooth area and the result of preprocessing.

$$
\Lambda(m, n) \in E(x, y)=\left|\begin{array}{cccc}
\lambda_{11} & \lambda_{21} & \cdots & \lambda_{m 1} \\
\lambda_{12} & \lambda_{22} & & \vdots \\
\vdots & & \ddots & \vdots \\
\lambda_{1 n} & \cdots & \vdots & \lambda_{m n}
\end{array}\right| \in\left|\begin{array}{cccc}
e_{11} & e_{21} & \cdots & e_{x 1} \\
e_{12} & e_{22} & & \vdots \\
\vdots & & \ddots & \vdots \\
e_{1 y} & \cdots & \cdots & e_{x y}
\end{array}\right|
$$

where $\Lambda$ is the entire tooth area, and $E$ is the tooth emphatic image in preprocessing. Third, the tooth ratio of tooth width to tooth image was calculated using an oral structure composed of four incisors, two cuspids, two premolars, and three molars on the upper and lower jaw. Figure 2 shows the calculation method and results. Then, teeth width per incisor, cuspid, premolar, and molar from each of the other tooth images is acquired. Teeth ratio was acquired to compare the entire tooth area with tooth size, and therefore, tooth sizes are assigned in the ratios of 5:3:2 per incisor, premolar, and molar, respectively [7].

Finally, three tooth areas were made using the acquired tooth ratio. Figure 2(c) shows the results of the division applied to the tooth ratio. $\Lambda^{f}$ is the incisor region, $\Lambda^{l}$ and $\Lambda^{r}$ are the side molar regions. Eq. (2) shows the relation between the entire tooth area and the three divided areas.

$$
\begin{aligned}
\Lambda(m, n) & =\Lambda^{l}(k, n)+\Lambda^{f}(j, n)+\Lambda^{r}(z, n) \\
& =\left|\begin{array}{cccc}
\lambda_{11}^{l} & \lambda_{21}^{l} & \cdots & \lambda_{k 1}^{l} \\
\lambda_{12}^{l} & \lambda_{22}^{l} & & \vdots \\
\vdots & & \ddots & \vdots \\
\lambda_{1 n}^{l} & \cdots & \cdots & \lambda_{k n}^{l}
\end{array}\right|+\left|\begin{array}{cccc}
\lambda_{11}^{f} & \lambda_{21}^{f} & \cdots & \lambda_{j 1}^{f} \\
\lambda_{12}^{f} & \lambda_{22}^{f} & & \vdots \\
\vdots & & \ddots & \vdots \\
\lambda_{1 n}^{f} & \cdots & \cdots & \lambda_{j n}^{f}
\end{array}\right|+\left|\begin{array}{cccc}
\lambda_{11}^{r} & \lambda_{21}^{r} & \cdots & \lambda_{z 1}^{r} \\
\lambda_{12}^{r} & \lambda_{22}^{r} & & \vdots \\
\vdots & & \ddots & \vdots \\
\lambda_{1 n}^{r} & \cdots & \cdots & \lambda_{z n}^{r}
\end{array}\right|
\end{aligned}
$$

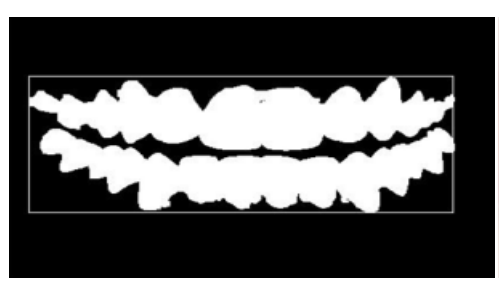

(a)

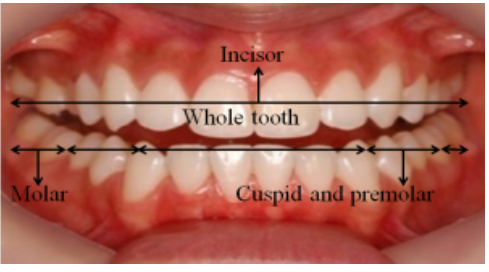

(b)

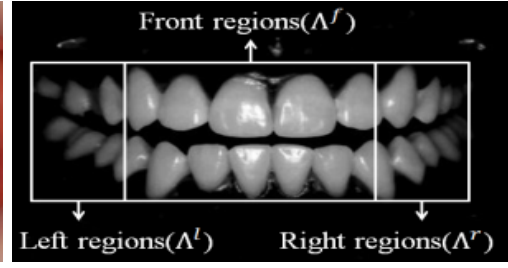

(c)

Fig. 2. Trisection method using tooth morphological characteristic, (a) whole tooth area, (b) each tooth size, (c) division result. 
where $n$ is the row of the image and $k, j$, and $z$ are the columns of the image.

The decrease in intensity occurs in the side molar regions. Therefore, enhancement is performed linearly on $\Lambda^{l}$ and $\Lambda^{r}$ using each pixel location per region and weight $\alpha$. Eq. (3) shows the enhancement method.

$$
\begin{aligned}
H & =\alpha \Lambda^{l}+\Lambda^{f}+\alpha \Lambda^{r} \\
& =\left(\sum_{k=d}^{1} \frac{k}{d}+\beta\right)\left|\begin{array}{cccc}
\lambda_{11}^{l} & \lambda_{21}^{l} & \cdots & \lambda_{k 1}^{l} \\
\lambda_{12}^{l} & \lambda_{22}^{l} & & \vdots \\
\vdots & & \ddots & \vdots \\
\lambda_{1 n}^{l} & \cdots & \cdots & \lambda_{k n}^{l}
\end{array}\right|+\left|\begin{array}{cccc}
\lambda_{11}^{f} & \lambda_{21}^{f} & \cdots & \lambda_{j 1}^{f} \\
\lambda_{12}^{f} & \lambda_{22}^{f} & & \vdots \\
\vdots & & \ddots & \vdots \\
\lambda_{1 n}^{f} & \cdots & \cdots & \lambda_{j n}^{f}
\end{array}\right|+\left(\sum_{r=1}^{r} \frac{r}{d}+\beta\right)\left|\begin{array}{cccc}
\lambda_{11}^{r} & \lambda_{21}^{r} & \cdots & \lambda_{z 1}^{r} \\
\lambda_{12}^{r} & \lambda_{22}^{r} & & \vdots \\
\vdots & & \ddots & \vdots \\
\lambda_{1 n}^{r} & \cdots & \cdots & \lambda_{z n}^{r}
\end{array}\right|
\end{aligned}
$$

Where $H$ is the enhancement result, $d$ is range of the side molar regions, and $\beta$ is the intensity control weight. Figure 3 shows the comparison before and after enhancement.

\subsection{Improved extraction method of watershed boundary and seed using trisection areas}

The watershed method as shown in Figure 4 was based on geographical method. The method creates pierced holds to form the catchment basins, and catchment basins are formed from pierced holes to dams, where a pierced hole and dam are seed and boundary in the watershed algorithm, respectively $[8,9]$. However, in accordance with the setup for seed and boundary in the conventional watershed, the dams and boundaries were constructed at bright parts and tooth borders, respectively. Consequently, boundaries were segmented between tooth and tooth. However, the method uses Sobel's edge and the

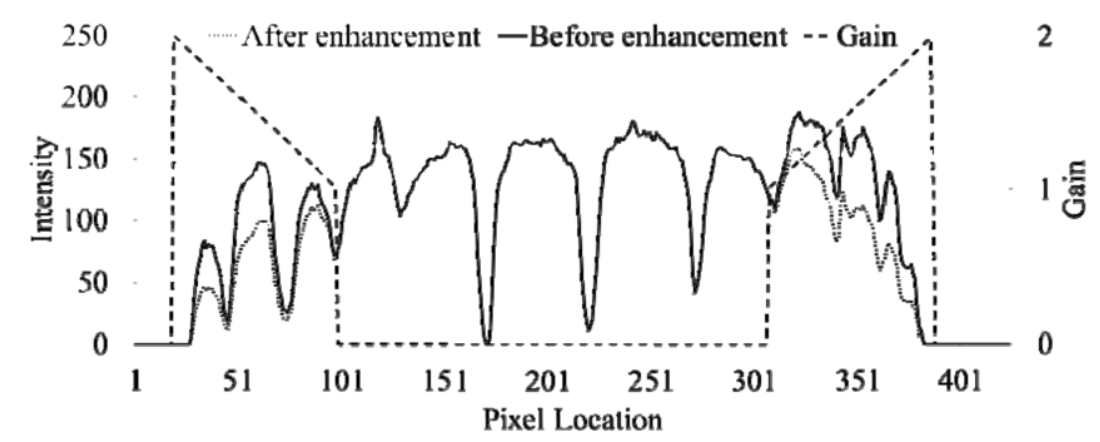

(a)

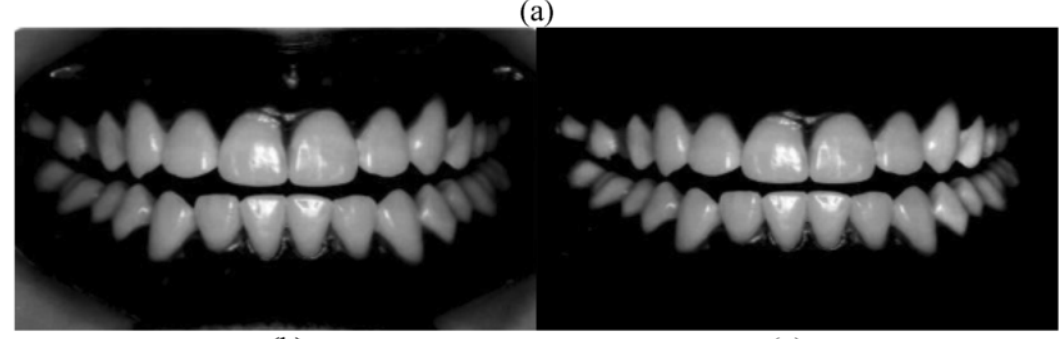

(b)

(c)

Fig. 3. Enhancement result, (a) image intensities before and after enhancement, (b) preprocessing result and (c) applied enhancement result. 
regional maximum value to address this disadvantage. In order to improve the problems of conventional method, complement image is used on the watershed algorithm, as shown in Figure 4(b). The extraction of boundary and seed is assignment method of different parameters at the each area. The boundary of watershed was made to assign each thresholding per area. First, Otsu's threshold method to the incisor region is applied. Second, half of the incisor threshold to the premolar region is applied. Finally, threshold value (25) to the molar region is applied. Then, each image was combined. Then, the boundary of obtained combined image was extracted for the boundary of watershed. The seed is acquired using the regional minimum value and morphology reconstruction. First, initial seed is acquired using the regional minimum value on the tooth complement image. Then, reconstruction is performed using the shrink and dilation morphology operation. Figures 4(c) and 4(d) show the resulting boundary and seed, respectively.

\section{Results}

Figure 5 shows the results of the conventional and proposed methods. Figures 5(a) and 5(c) using the conventional method reveal problems of non-detection in the molar region and over- and overlapsegmentation of the incisors owing to Sobel's edge characteristic and specular reflection. Figures 5(b) and 5(c) utilizing the proposed method show improved tooth detection in the molar region, and increased accuracy.

The accuracy of the proposed method was verified by applying it to each of the other images. Table 1 shows the comparison results of the conventional and proposed method. The proposed method detects all the teeth in the molar regions and has no over-or overlap-segmentation due to specular reflection. Teeth number 4 and 5 show that the conventional method has problems in detecting tooth number because of preprocessing that emphasizes the white parts of the tooth. Therefore, non-detections occur owing to the difference from normal tooth color or a prosthetic tooth. Figure 6 shows that the proposed

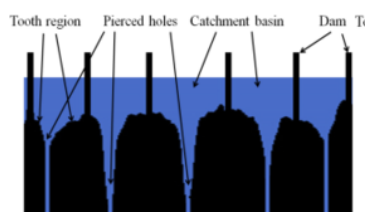

(a)

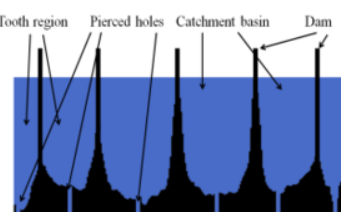

(b)

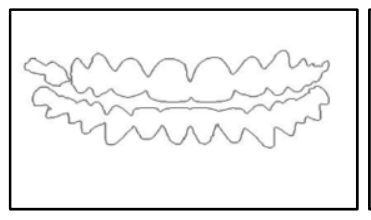

(c)

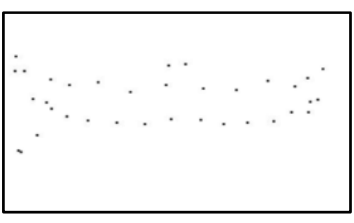

(d)

Fig. 4. Watershed algorithm principle of, (a) conventional watershed method, (b) complement watershed method, (c) extracted boundary of watershed and (d) extracted seed of watershed.

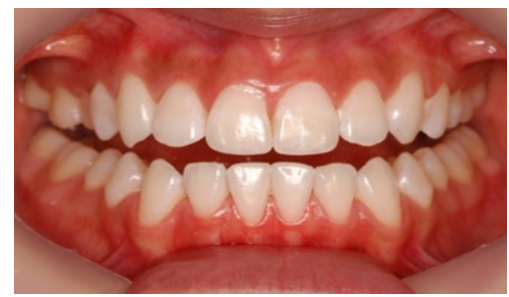

(a)

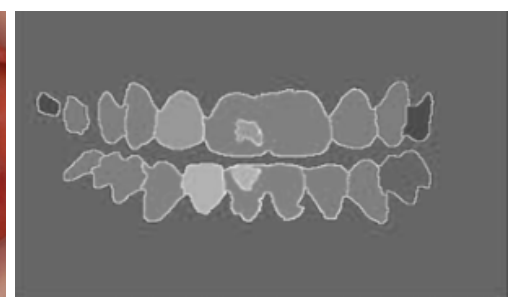

(b)

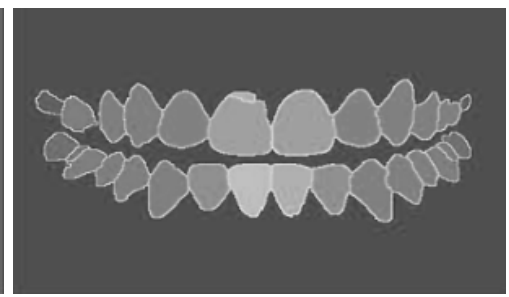

(c)

Fig. 5. Tooth segmentation result, (a) original image, (b) conventional method segmentation and (c) proposed method segmentation. 
method better prevents error rate than the conventional method. However, teeth numbers 3,6 , and 7 show partial over-segmentation due to specular reflection that is higher than that for other teeth or similar entire tooth intensities.

In summary, Table 1 shows that the proposed method performs better than the conventional one in terms of overall detection ratio and suppression of over- and overlap-segmentation.

\section{Conclusion}

The watershed algorithm is suitable to segment the tooth image, but has disadvantages of less accuracy in detecting tooth. In order to improve the problems, the trisection method was proposed, which is a division method to tooth areas considering teeth width.

Our results show that the proposed method performs better than the conventional method. Therefore, the proposed method was expected to simplify the inconvenience of prosthetic appliance manufacture complexity and improve the location information efficiency for orthodontics by tooth shape.

Table 1

Results of tooth detection by conventional marker controlled watershed algorithm method and proposed method

\begin{tabular}{|c|c|c|c|c|c|c|c|}
\hline & \multirow{3}{*}{$\begin{array}{l}\text { Number } \\
\text { of teeth }\end{array}$} & \multicolumn{3}{|c|}{ MCWA method } & \multicolumn{3}{|c|}{ Proposed method } \\
\hline & & \multirow{2}{*}{$\begin{array}{l}\text { Detected } \\
\text { tooth }\end{array}$} & \multicolumn{2}{|l|}{ Error } & \multirow[b]{2}{*}{ Detected tooth } & \multicolumn{2}{|l|}{ Error } \\
\hline & & & Over-seg. & Overlap-seg. & & Over-seg. & Overlap-seg. \\
\hline Total & 599 & 510 & 137 & 26 & 532 & 52 & 30 \\
\hline Average rate [\%] & - & 86.39 & 28.85 & 5.28 & 90.3 & 10.6 & 5.14 \\
\hline
\end{tabular}

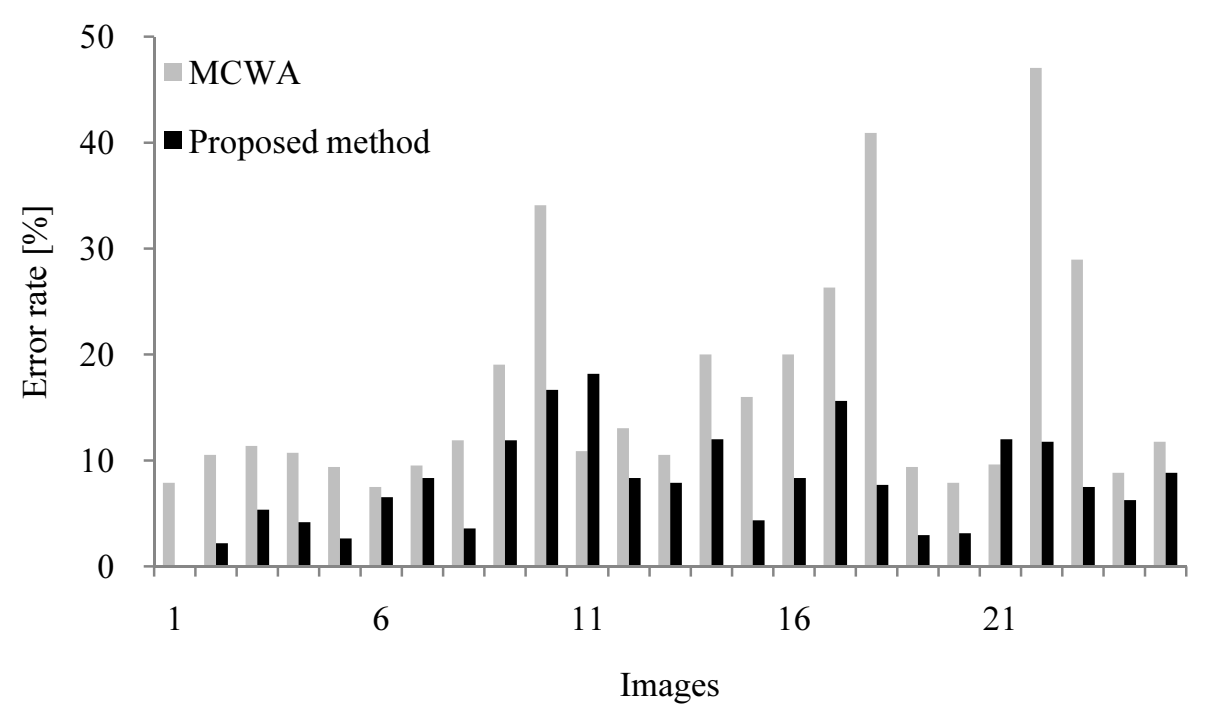

Fig. 6. Error rate of MCWA and proposed method. 


\section{Acknowledgement}

This research was financially supported by the Ministry of Knowledge Economy (MKE), Korea Institute for Advancement of Technology (KIAT) and Dae-Gyeong Leading Industry Office through the Leading Industry Development for Economic Region, and the "Over regional linked 3D convergence industry promotion program" through the Ministry of Trade, Industry \& Energy (MOTIE) and Korea Institute for Advancement of Technology (KIAT).

\section{References}

[1] M. Rema and S.N. Madhu, Segmentation of human intestinal parasites from microscopy images using localized meanseparation based active contour model, Biomedical Engineering Letters 3 (2013), 179-189.

[2] D. Hwang and G.L. Zeng, Special issue on medical imagen, Biomedical Engineering Letters 4 (2014), 1-2.

[3] G.-S. Lee and K. Park, Development of automatic scanning system for intra-oral scanner using SCARA robot, Proc. the Society of CAD/CAM Conference, 2013, 332-337.

[4] F. Ullah, G.-S. Lee and K. Park, Development of a real-time 3D intraoral scanner based on fringe-projection technique, Transactions of the Society of CAD/CAM Engineers 17 (2013), 156-163.

[5] Y.Q. Zhao, Q.P. Zhao and A.M. Hao, Multimodal medical image fusion using improved multi-channel PCNN, Biomedical Materials and Engineering 24 (2014), 221-228.

[6] S.-T. Lee, K.-S. Kim and T.-H. Yoon, Individual tooth image segmentation by watershed algorithm, The Trans. KIEE 59 (2009), 210-216.

[7] R.C. Sceid and G. Weiss, Woelfel's Dental Anatomy, Wolters Kluwer, Philadelphia, 2012, 110-143.

[8] G. Wood, Digital Image Processing Using MATLAB, Prentice-Hall, New Jersey, 2004, 567-636.

[9] Q. Wu, F.A. Merchant and K.R. Castleman, Microscope Image Processing, Academic Press, Massachusetts, 2008, 159194. 